\title{
Fuzzy Logic-Based Control for Autonomous Vehicle: A Survey
}

\author{
Ishaya Emmanuel ${ }^{*}$
}

\author{
Usmanu Danfodiyo University Sokoto, 84004, Nigeria.
}

\begin{abstract}
Fuzzy set, since its advent has played an important role in control systems and many other area of applications. One of such area is the control of autonomous vehicle. There seem to be some difficulty however, for a new timer trying to get a clear picture of the autonomous navigation problem. To this end, this survey presents a panoramic view of the Intelligent Transportation Systems with some few example of the Advance Driver Assistance Systems and a good discussion on the autonomous systems with its eminent problems. More attention was focused on the fuzzy controllers designed for collision avoidance; as its performance has largely simplified and smoothens the collision avoidance process of an autonomous vehicular system.
\end{abstract}

Index Terms: Fuzzy Logic, Autonomous Vehicle, Robot, Obstacle Detection, Collision avoidance.

(C) 2017 Published by MECS Publisher. Selection and/or peer review under responsibility of the Research Association of Modern Education and Computer Science.

\section{Introduction}

From the very onset, researchers have sort for ways to replace the human operator, that is pliable and susceptible to errors, with some sort of machinery -that is less prone to error, effective, efficient, and never complains of tiredness due to monotonous routine. It is as a result of this quest the technological community has experienced a revolution taking forms like: at its primitive stage, implementation of devices that aid simple calculation to a more complex stage that constitutes the application of machinery and machine systems for industrials production processes; this also entails the use of robotics for surgical procedures $[1,2,3]$ and autonomous devices for planetary explorations. More recently, these technological advancements resulted to the implementation of simple but powerful household [4] robots for domestic chores like laundry, vacuum and dishing to a more complex task handling robots like the mobile nurse [5] implemented to assist bedridden patients.

Another interesting aspect of this revolution is the implementation of vehicular systems capable of driving along country and urban settings $[6,7,8]$ without human aid of whatsoever form. Nowadays, virtually every process can be automated by some intelligent machine and controllers as evident in [9] which reported that the

Corresponding author.

E-mail address: 
International Federations of Robotics concluded that there have been a $19 \%$ increase in the use of robots with a statistics of 86,200 to 106,300 units in just a space of three years -2004-2007. The aforementioned revolution gave birth to an art (Intelligent Transport Systems) which focuses on the implementation of Advance Driver Assistance Systems (ADAS) and Automatic Ground Vehicle (AVD) as some call it, or Unmanned Ground Vehicle (AVG) as others see it. Whichever name it is called, the idea or rationale is to design and implement a vehicular system capable of simulating the human driving operation and do it very well. The need to implement ADAS and AVG boils down to the fact that, since human drivers' factors are responsible for about $95 \%$ of road accidents $[10,11]$, which can be attributed to the human susceptibility pointed earlier, it is believed however, that a well programed autonomous vehicle should outperforms the human drivers; or better still, a set of warning systems can assist the driver with promptings of potential collision or information about the environment. This has been the goal and a successful one (to some extent) since report [13] has it that the use of ADAS (Adaptive Cruise Control- ACC) considerably reduced the chances of vehicle collision. Many ADAS systems have been proposed and some implemented in the top class cars of some automakers. Few of these are: Adaptive Cruise Control System that helps to keep reasonable distance between front cars and the ADAS aided system by regulating vehicular speed; [11] presents a rich survey on Intelligent Collision Systems and ACC; Lane Detection System [16] helps maintain track by detecting the road lanes; speed bumps detector [15]; potholes detectors [16, 17, 10]; Overtaking Maneuver Systems [18]; Car Parking System, Terrain Classification System [19, 20, 21, 8], etc. Although, the ADAS systems have considerably helped the driving process, however, the need to implement a fully autonomous vehicle cannot be undermined since its use has gained grounds in medical and military reconnaissance, exploration of terrains yet safe for humans, media report in war infested zone, etc. As such, efforts have been channeled to the design and implementation of autonomous vehicle by researchers from all around the world. To serve as incentive to researchers in the art, challenges have been organized in different countries aimed at exploring a given navigation problem. Example of such is the DARPA Urban Challenge won by [22], The Vehicle Following Problem, etc. Other problems explored in the art are: Lane and Wall Following Problems, Search and Rescue Robot implementation, Children Cone Play Simulation Problem, etc. These have gone a long way in the automation of navigation process as there now exists sophisticated but easy to use sonar, range finders and cameras; development of libraries (e.g. OpenCV) and API's (Application Programming Interface) that ease the robot's implementation process; and design of fast image processing schemes [52]; miniaturized rovers and mechatronic/mini robots' devices for prototyping, etc. However, much work is still in progress with the aim of making the autonomous vehicle capable of working in real-timing with high speed. It is along this line of thought this review focuses on presenting an overview of the autonomous vehicle system with few literatures in the art as there exist tons of published papers on this subject. This work also presents brief discussion on the fuzzy logic-based controllers which stands as the heart of this peer review. The paper is organized thus: the following section ushers an overview of autonomous vehicle, followed by section three - discuses fuzzy control systems, next is section four -presents some fuzzy controllers, and section five concludes the work.

\section{Autonomous Vehicle}

Autonomous vehicle design and implementation started gaining attention in the research community around late 80's and early 90's [11], this may be attributed to the fact that their use has gained ground in medicine, military reconnaissance, industrial applications, domestic appliances, touring guide etc. Albeit any area autonomous robot has been employed, its fundamental operation is to move from one part to another while recognizing obstacle and providing means for collision avoidance in the process. This however, boils down the navigation process into obstacle detection and collision avoidance, hence the two major problems in the art. For an autonomous vehicle to successfully accomplish the task of moving from one point to another, it needs to have the mechanism that enables it know what the environment looks like. This implies the use of vision systems that provide the autonomous robot with the ability to detect obstacle and act befittingly. Although, other means that requires the use of model representation of the world, as will be seen shortly, are in place for 
obstacle-free path planning, however, a vision system enables the robot to better thrive in an environment characterized with uncertainties -an inherent characteristic of the urban or country settings. This vision system consists of sensors and image processing algorithms for obstacle detection; examples of some sensors often used are:

- $\quad$ RADAR: radio detecting and ranging e.g. sonar, ultrasonic sensors, etc.

- $\quad$ LIDAR: Light Detecting and Ranging e.g. IR sensor,

- Stereo imaging: the use of either two or single camera for acquiring a 3D or 2D image representation of the environment respectively.

It is noteworthy herein that while the use of Stereo imaging systems gives more information about the environment, it however requires complex calculations that often constrains the real-time operation of the robot [12]. On the other hand, sonars are fast in operation and simple to implement even though their use is restricted to ranging and obstacle pinging without any information about the environment, except maybe in case of multisensor systems as would be seen shortly. Operating concurrently with the obstacle detection system is the collision avoidance mechanism that bespeaks the robot's ability to either stop when an obstacle is pinged or employ some control decision to detour around the obstacle. Devices that aid the avoidance process are controllers -these make decision, and actuators that perform the task. Subsequent section shed more light on controllers, for now, examples of some actuator are steeper motors, DC motors, servo, etc. interested readers should consult [25] for more examples of actuators. The obstacle detection and collision avoidance subsystems makes up the autonomous vehicle system, hence the subsequent subsection presents brief review of these.

\section{i. Obstacle Detection}

Some classifications exist for obstacle detection systems, one of which, is the Qualitative and Quantitative obstacle detection approach proposed by Zhang et al. [25]. By qualitative, the authors meant, obstacle detection system whose results hinge on the presence or absence of obstacle without further information; in this vein, they proposed the Known Ground Plane and the Unknown Ground Plane algorithms for obstacle detection. On the quantitative side, they proposed a third algorithm that computes a partial 3D structures of the obstacle. Polarizing proposed literatures according to this categorization for obstacle detection in the art, [26, 25] polarizes to the qualitative obstacle detection approach and the schemes found in $[27,25,19,7,23,28]$ categorizes as the quantitative obstacle detection approach. Another interesting concept is the Multi-sensor approach for obstacle detection. Systems deployed with this approach constitutes the use of different sensors (radar, stereos, sonar) for obstacle detection. This approach also introduces the concept of data/sensor fusion architecture whereby all information from the sensors are fused to produce inputs to the control systems. [6, 8, 29] are some of the literatures that explored this approach.

\section{ii. Collision Avoidance}

$\mathrm{Xu}$ et al. [30] categorizes the approaches employed to solve the navigation control problem into: modelbased method and fuzzy logic with the neural network reactive based methods. Interested readers can consult $[31,32]$ for more on the model based method of collision avoidance schemes. Fuzzy logic and Neural network reactive has been combined for a fine, robust and adaptive control, however, fuzzy logic, by itself, can achieve a fast and considerably smooth control as seen shortly. Although difficult to place under any of the subsections, mobile robot's navigation process classification suggested in [33] on autonomous robot need some audience. The authors presented three categorization proponents in the art employed in recent times. First is the model based approach in which the robot maintains a copy of the world's model for obstacle free path planning. [5] applied this method. Sensor based approach [10, 30, 34], which emphasizes only the use of sensors information about the environment for navigation decision. And last on the list is Hybrid approach. Systems [35, 5] 
deployed with this

approach combine both model and sensor information for autonomous navigation process. Model in this concept is not restricted to the environment's map on which the autonomous vehicle operates, but is extended to accommodate both goals and target-based approaches.

\section{Fuzzy Logic Control Systems}

Fuzzy logic, since its advent [36] has assumed an important role in control systems; not only in control systems has fuzzy set (as was called at its birth) gain grounds, its use has found place in many areas as presented in [37]. Fuzzy logic was first employed for the control system of a steam engine reported in [38], Hombland and Ostergard presented the first application of fuzzy logic controller in an industrial process for a cement kiln [43]. Nowadays, consumer product's (vacuum cleaners, air conditioner, washing machine, etc.) controls are programed with calculus of fuzzy rules (CFR) that tend to increase the Machine Intelligent Quotient [39] by modeling the human expertise using linguistic variables that conceptualizes the fuzziness in human decision making. Unlike conventional controls that often require lot of time and high computational cost, fuzzy logic conceptualizes the human operational experience -of an operator -into a controller that comprises of IF/THEN rules which simulates the operators "know how" in an algorithmic manner made up of fuzzy sets [40]. The ease with which this can be done results to a fall in the cost of consumer products. Fuzzy control systems, unlike expert systems, are made up of four parts thus: the fuzzifier -this convert crisp input variables to linguistic term in a process known as fuzzification; the rule base houses the IFTHEN rules; the inference module employs if-then rule on inputs to infer the control action as would be done by the human operator; and the defuzzifier is responsible for converting the results into crisp output in a process called defuzzification. Figure 1 [41] depicts a general fuzzy controller with the fundamental processes, however, many methods have been put in place for these process. Example is the MinMax, Centroid or Center of Gravity method used for defuzzification [42]. Fuzzy control systems (FCS) are classified into two types namely: model-free and model based FCS [44]. When compared with its equivalent conventional PID (Proportional Derivative-Integral) control systems, model-free FCS's show a better performance, which may be accrued to the fact that a lot of work have been directed toward its development. On the other hand, model based fuzzy comprises of Type-1 FCS, Type-2 FCS and Type-3 FCS [45]. Readers interested on getting more insight on the concept of fuzzy control systems can visit [45], this survey contains a comprehensive knowledge on the fuzzy control system and its industrial application that spans from manufacturing, servo controls, robotics, up to automotive. For light on the emerging Hybrid systems -fuzzy logic plus soft computing methodology e.g. neural network, genetic algorithm etc. -readers can consult [46]. Others who may wish to explore fuzzy logic networked systems can check [44] as the authors have done justice to the subject and also provided future trends to help researchers sail with the tide. This paper would be lacking if the brilliant work done in [37] is not lay bare; as it presents a well packed survey on the fuzzy software systems in virtually all the application areas of fuzzy logic which include GIS, image processing, handwriting recognition, etc.

\section{Fuzzy Controllers for Autonomous Vehicle}

As evident in the previous section, fuzzy controllers, due to their higher speed and smooth control [47], are better option to tackle the real-time issue [32] of obstacle detection and avoidance control in autonomous navigation. As such, this section presents selected literatures that employed fuzzy algorithm in the art. However, this review article presents contributions from different literatures without any critique of whatsoever to the researchers' effort. Beom and Cho [34] proposed: A Sensor Based Obstacle Avoidance Controller for a mobile robot using Fuzzy Logic and Neural Network. Herein, the implemented robot (LCAR), equipped with a set of eighteen ultrasonic sensors, uses neural network (aka Situation Classifier) to estimate the obstacle(s) position from range image; the distance to the obstacle, heading angle and obstacle direction constitute the fuzzy input variables with increasing steering angle and velocity as the outputs. Xu et al. [30] employed fuzzy logic for real- 
time reactive control in navigating an intelligent autonomous robot in a changing environment having multiple static obstacles of different form. Fuzzy logic was used to navigate an obstacle away from trap and endless loop -the local trapping and oscillating wandering problem -by using the new virtual target location, obtained from the "local target switching scheme", and the perspective distances of the obstacle to steer and accelerate the wheel and steering of the mobile robot (Nomad 200). Hodge and Trabia [48] proposed a "Steering Fuzzy Logic Controller for an Autonomous Vehicle" that simulates the human reasoning approach for avoiding obstacles. The scheme separates the obstacle avoidance process into a set of task handled by modules implemented as fuzzy controllers.

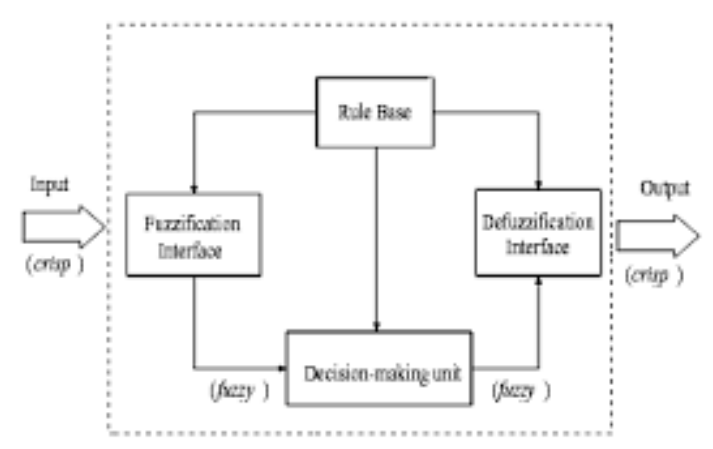

Fig.1. Basic Configuration of a Fuzzy Logic System

First is the driving task that further consist of target steering plus obstacle avoidance steering; next is the bug steering module that handles the problem of trapping and wandering, and last is the module that handles the vehicles orientation in respect to its goal. In all cases, except the orientation controller, appropriate inputs (steering angle, target angle and obstacle's distance) are fuzzified to yield corrected steering angle. Narouzi et al. [47] improve their previous work -Recursive Line Extraction Algorithm from 2D Laser Scanner Applied to Navigating a Robot -by proposing a fuzzy controller for the navigation scheme implemented on a search and rescue robot (NAJI V). The fuzzy controller decides path using lines and fusion data obtained from Lasar and sensors. In practice, the Lasar scanner provides the fuzzy controller with distance and angle of obstacle for command generation. On the contrary, if the scanner information contains obstacle and wall, sonar information is combined with obstacle distance for navigation. Depending on the output membership function, eight fuzzy rules assigns positive or negative (forward or backward) speed to the wheels, hence by concurrent assigning values to the speed and steering angle, the robot is able detour an obstacle without stopping. Gardeazabal et al. [50] combined image processing and Type-I fuzzy algorithm to implement an obstacle avoiding mobile robot which uses information (i.e. object distance and attack angle) from a stereo vision camera to generate fuzzy rules that control the collision avoidance process. The proposed fuzzy controller processes the object distance and the attack angle to yield a tuned velocity for the robot's navigation along its preprogrammed circular trajectory. Baasandorj et al. [50] implemented a path planning and obstacle avoidance algorithm using Modal Predictive Control and fuzzy logic in a rather complex environment consisting of static and mobile obstacles. The scheme applied fuzzy logic to control obstacle detection and determines the robot's orientation. Ultrasonic sensors provide the distance and angle, of the nearest obstacle in relation to the goal, these represent the inputs and the heading angle as the output. Bounini et al. [14] in their work, "Autonomous Vehicle and Real Time Lanes Detection and Tracking", applied fuzzy rules to maintain the position of vehicle at the center of the right lane. In operation, the fuzzy controller generates steering angle in relation to the vehicles direction that keep and track the center of road lanes. Cao and Hall [33] employed command fuzzy technology in a hybrid approach for the navigation of an autonomous guided rover (BEARCAT). With the aid of CCD cameras and Plaroid sensors, BEARCAT was able to detect lanes for maintaining track and to detect obstacle respectively. 
Center of gravity defuzzification method defuzzifies output from each behavioural controllers. Maslak and Butkiewiez [51] presented sonar based approach for autonomous navigation vehicle equipped with a pair of GPROA21 distance IR (Infrared Sensor), DFRduino motor shield and Arduino Uno microcontroller for obstacle sensing and collision avoidance actuation. A five fuzzy rule controller having distance as input represented with three linguistic variables and angle of drive as output was design for the collision avoidance process. The authors converted angle of drive into differential drive speed on the wheels thereby allowing the vehicle to detour obstacle. Thongchai and Kawamura [42] implemented a behavior based Multi-sensor obstacle detection and avoidance robot (HelpMate) designed with a set of behaviors that ranks from the highest to lowest thus: task oriented -divides into goal and wall following, obstacle avoiding and emergency behavior. A fuzzy controller module was designed for each behavior having inputs (distance of obstacle) from all sonar represented using Gaussian membership function. Method of centroid defuzzifies outputs (angular velocity) for HelpMate's navigation. Claudia et al. [12] proposed a simple image processing; and Gaussian-noise robust algorithm using 3D depth sensors (Xtion Pro Live) for obstacle(s) detection. The proposed algorithm employs Kolmogorov-Smirnov test for image processing, these yields obstacle's distance and angle fed to a scalable fuzzy controller which, using fine linguistic variables, generates steering angle and wheel speed for collision avoidance. Gazebo Simulator was used to validate the proposed scheme. It is noteworthy at this juncture that fuzzy logic is not constrained to the implementation of control logic for autonomous robot, it has also been used in the obstacle detection process. Example is the work of Zoubir and Abdelnaheb [52] who employed fuzzy rules for the fast detection of edge in stereo images processing. Cabreira et al. [53] also used fuzzy obstacle detection function, constantly called to estimate the closeness of obstacle to the robot in the path selected by a genetic algorithm. As stated earlier, this work only presents few literatures in the art, however the literature contained herein can set a foundation for a starter in the art.

\section{Conclusion}

This work started by presenting a background to the autonomous navigation problem for the unmanned ground vehicle; this further enumerate some ADAS systems which is another good research area for those interested in intelligent transportation systems. It continued by bringing to spotlight the need for vehicles to be fully autonomous notwithstanding the many ADAS systems already in play in the automobile industries. A detailed description of the autonomous problem was discussed with different classification of the methods/approaches employed to address the obstacle detection and avoidance problem. This featured the qualitative and quantitative obstacle detection algorithms. As for the collision avoidance problem, the conventional model-based methods consisting PI and PID approach was juxtaposition with the fuzzy logic plus neural network reactive based approach. A whole section was dedicated to fuzzy logic control system's review, and pointers where placed to direct curious readers to some well-nourished survey papers that are sure to solidify readers' knowledge of the concept of fuzzy logic in various areas of application, and introduces the vast materials, software, models and API's available for the implementation of fuzzy systems. The author at this point may wish to make clear the fact that some interesting issue -like the multi-obstacle environment that often leads to trapping and oscillating/wandering which is often an issue inherent to target or goal-based approach has not been considered. Also, this work paid no attention to newer trend of multi-agent collision avoidance system; networked obstacle detection and avoidance systems, and the concept of Depth Sensing. Albeit, the information contained herein can however set the ball rolling for a starter in the art.

\section{References}

[1] Z. Hong, Y. Chao, and Z. Lei: A MRI guided robot for neurosurgery: Design and control. Proceedings of the IEEE International Conference on Information and Automation, 2008, 1, pp 759-764.

[2] S. J. Song, Y. Moon, D. H. Lee, C.B. Ahn, Y. Jo and J. Choi: Comparative Study of Fuzzy PID Control 
Algorithms for Enhanced Position Control in Laparoscopic Surgery Robot, Journal of Medical and Biological Engineering February 2015, Volume 35, Issue 1, pp 34-44.

[3] Qinjun, D.: Fuzzy PID control orienting medical robot in minimally invasive surgery. Proceedings of the IEEE International Conference on Automation and Logistics, 2007, 1, pp 2633-2638

[4] N. Wakami: Fuzzy control and neural networks: Applications for consumer products, Fuzzy Logic and Fuzzy Control Volume 833 of the series Lecture Notes in Computer Science, August 2005, pp 97-105.

[5] J. Borenstein and Y. Koren: Obstacle Avoidance with Ultrasonic Sensors. International Journal of Robotics and Automation, Vol 4, No.2 April 1988. pp 213-218.

[6] C. Stiller, J. Hipp, C. Rossing and Ewald: Multisensor Obstacle Detection and Tracking. Image and Vision Computing 18 (2000). pp 389-396.

[7] N. Kehtarnavaz, N. C. Griswold and J. S. Lee: Visual Control of an Autonomous Vehicle (BART) -The Vehicle-Following Problem, IEEE Transaction on Vehicular Technology, Vol. 40, No. 3, August 1991. Pp 654-622.

[8] D. C. Carl, D. G. Armstrong, A. Maryum, S. Solanki, D. MacArthur, E. Zawodny, S. Gray, T Petroff, M. Grifis and C. Evans: Development of an Integrated Sensor System for Obstacle Detection and Terrain Evaluation to Unmanned Ground Vehicle. In Proc. of SPIE Vol. 5804, pp. 156-165.

[9] A. M. Adil and U. F. Aziz: Sonar Based Obstacle Detection and Avoidance Algorithm, in IEEE International Conference on Signal Acquisition and Processing, 2009, pp 98-102.

[10] S. S. Rode, S. Vijay, P. Goyal, P. Kulkami and K. Arya: Pothole Detection and Warning System: Infrastructure Support and System Design, in IEEE international conference on electronic computer technology, 2009, pp 286-290.

[11] A. Vahidi and A. Eskandarian: Research advances in Intelligent Collision Avoidance and Adaptive Cruise Control. in IEEE Transactions on Intelligent Transportation Systems, Vol. 4, No. 3, September 2003. Pp 143-153.

[12] A., Claudi, D., Accattoli, P., Sernani, P., Calvaresi and A. F., Dragoni: A Noise Robust Obstacle Detection Algorithm for Mobile Robots Using Active 3D Sensors, 56th International Symposium ELMAR-2014,, Zadar, Croatia, September 2014, pp 91-94.

[13] A. Touran, M. Brackstone, and M. McDonald, A collision model for safety evaluation of autonomous intelligent cruise control, Accident Analysis and Prevention, vol. 31, May 1999, pp. 567-578.

[14] F. Bounini, D. Gingras, V. Lapointe and H. Pollart: Autonomous Vehicle and Real Time Road Lanes Detection and Tracking, in IEEE Proc. VVPC, 978-1-4673-7637-2/15.

[15] G. A. Kiran and S. Muralis: Automatic Hump Detection and 3D View Generation from a Single Road Image. In Proc. IEEE international Conference on Advances in Computing, Communications and Informatics (ICACCI), 2014. pp. 2232-2283.

[16] X. Yu and E. Salaris: Pavement Pothole Detection and Severity Measurement Using Laser Imagin text, (2011).

[17] A. Mednis, G, Strazdins, R. Zviedris, G. Kanonirs and L. Selavo: Real Time Pothole Detection using Android Smartphones with Accelerometer, in IEEE of DCOSS, 2011.

[18] A. D. Jarnea, R. Dobrescu, D. Popescu and L. Ichim: Advance Driver Assistance System for Overtaking Maneuver on a Highway, in IEEE 15th International Conference on System Theory Control and Computing, Romania, October 2015. pp 759-764.

[19] R. Manduchi,A, Castano, A.Talukderand L. Matthies: Obstacle Detection and Terrain Classification for Autonomous Off-Road Navigation. Autonomous Robot 18, of Springer Science, Netherland, 2005. pp. 81-105.

[20] Y. Cheng-peng and Y. Xian: Terrain Classification for Autonomous Navigation using Lasar Sensing. in IEEE of the 1st International Conference on Information Science and Engineering (ICISE) 2009, pp. 14571470.

[21] M. Bajracharya, B. Tang, A. Howard, M. Turmon, and L. Matthies: Learning Long-Range Terrain Classification for Autonomous Navigation, in IEEE International Conference on Robotics and Automation, 
Pasadena, CA, USA, May 2008, pp 40184024.

[22] M. S. Darms, P. E. Rybski, C. Baker, and C. Urmson: Obstacle Detection and Tracking for the Urban Challenge, in IEEE Transactions on Intelligent Transportation Systems, VOL. 10, NO. 3, September 2009, pp 475-484.

[23] A. Talukder, R. Manduchi': A. Rankin and L. Matthies: Fast and Reliable Obstacle Detection and Segmentation for Cross-country Navigation, in IEEE of IVS, 2002, pp 610618.

[24] Artificial Intelligence: Intelligent Systems, www.tutorialspoint.com.

[25] Z. Zhang, R. Wiess and A. R. Hanson: Obstacle Detection Based on Qualitative and Quantitative 3D Reconstruction, in IEEE Transactions on Pattern Analysis and Machine Intelligence, Vol. 19, No. 1, 1997. pp 15-26.

[26] D. Nair and J. K. Aggarwal: Moving Obstacle Detection from a Navigating Robot, IEEE Transactions on Robotics and Automation, VOL. 14, NO. 3, June 1998, pp 404-416.

[27] K. Storjohann, Th. Zieke, H. A. Mallotand W. von Seelon: Visual Obstacle Detection for Automatically Guided Vehicle, IEEE1990, pp 761-766.

[28] H. Zhang, Y. Jia, N. Xi and A. Song: Obstacle Avoidance for Mobile Manipulation by Real-Time SensorBased Redundancy Resolution, in Proc. of IEEE International Conference on Robotics and Biometrics December 2012, Guangzhou, China, pp 2369-74.

[29] R. Sosa and G. Velazquez: Obstacle Detection and Collision Avoidance System Developed with Virtual Model, in Proc. in IEEE ICVES 2007, pp 1-8.

[30] W. L. Xu, S. K. Tso, and Y. H. Fung: Sonar Based Reactive Navigation of a Mobile Robot through Local Target Switching, in IEEEICA, Montercy, 1997, pp 361-366.

[31] J. Borenstein and Y. Koren: Real Time Obstacle Avoidance for Fast Mobile Robot in Cluttered Environment, IEEE 1990, pp. 572577.

[32] R. Lagisetty, N. K. Philip, R. Padhi and M. S. Bhat: Object Detection and Obstacle Avoidance for Mobile Robot Using Stereo Camera, in IEEE international conference on control Applications (CCA), Hyderabad, India, August 2013, pp. 605-610.

[33] M. Cao and E. Hall: Fuzzy Logic Control for an Automated Guided Vehicle, Center for Robotics Research University of Cincinnati, Cincinnati, OH 45221.

[34] H. R. Boem and H. S. Cho: A Sensor Based Obstacle Avoidance Controller for a Mobile Robot using Fuzzy Algorithm and Neural Network, IEEE 1992, pp. 1470-1475.

[35] B. I. Hartman, Y. Kanayama and T. Smith: Model and Sensor Based Precide Navigation by an Autonomous Vehicle, Advance Robotics, Springer-Verlag Berlin Heidelberg, 1989, pp 98-109.

[36] L. A. Zadeh: Fuzzy Sets. Information and Control, 1965, pp 338-353.

[37] J. Alcala-Fdez and J. M. Alonso: A Survey of Fuzzy Systems Software: Taxonomy, Current Trends and Prospects, Article in IEEE Transactions of Fuzzy Systems, February 2016.

[38] E. H. Mamdani and S. Assilian: An Experiment in Linguistic Synthesis with a Fuzzy Logic Controller, International Journal of Man-Machine Studies July 1975, pp 1-13.

[39] E. Cox: The Fuzzy Systems Handbook -A Practitioner's Guide to Building, Using, and Maintaining Fuzzy Systems, Academic Press, Incorporation 1994.

[40] W.J.M. Kichert and E.H. Mamdani: Analysis of a fuzzy Control Systems, Fuzzy Sets and Systems, vol.1, no. 1 , pg. 29-44, 1978

[41] http://www.scielo.br/img/revistas/ca/v14n4/a05fig01.gif.

[42] S. Thongchai and K. Kawamura: Application of Fuzzy Control to a Sonar-Based Obstacle Avoidance Mobile Robot, in Proc. of IEEE International Conference on Control Applications, Anchorage, Alaska, USA September 2000, pp 425-430.

[43] L. P. Holmblad and J. J. Ostegard: Control of a Cement Fuzzy Kiln by Logic, in Fuzzy Information and Decision Processes, (M.M. Gupta and E. Sanchez, eds), North-Holland, Amsterdam, 1884.

[44] Y. Luo, Z. Wang, G. Wei, B. Shen, X. He, H. Dong and J. Hu: Fuzzy-Logic-Based Control, Filtering, and Fault Detection for Networked Systems: A Survey. Mathematical Problems in Engineering, Hindawi 
Publishing Corporation, Vol 2015.

[45] R. E. Precup and H. Hellendoorn: A Survey on Industrial Application of Fuzzy Control, Computers in Industries, 2011, Vol. 62 no. 3, pp 213-216.

[46] N. Walia, H. Singh andA.Sharma: ANFIS: Adaptive Neuro-fuzzy Inference System -A Survey, International Journal of Computer Applications, Vol. 123 no. 13 August 2015. pp 32-38.

[47] M. Norouzi, A. Karambaskhsh, M. Namazifar and B. Savkovic: Object Obstacle Based Navigation of Mobile Robot with Obstacle Avoidance using Fuzzy Controller, IEEE International Conference on Control and Automation, Christchurch, New Zealand, December 2009, pp.169-174.

[48] N. E. Hodge and M. B. Trabia: Steering Fuzzy Logic Controller for an Autonomous Vehicle, Proc. of IEEE International Conference on Robotics and Automation, Detroit, Michigan, May 1999, pp 2482-88.

[49] D. Gardeazeaball, V. Ponomaryov and I. Chairez: Fuzzy Control for Obstacle Avoiding in Mobile Robots Using Stereo Vision Algorithms, in ICEEE, 2011. pp xxx.

[50] B. Baasandorj, R. Reyaz, P. J. Ho, C. W. Cheol, D. J. Lee and K. T. Chong: AMobile Robot Obstacle Avoidance Using Fuzzy Logic and Model Predictive Control. Applied Mechanics and Materials, Vols. 548-549 (2015) pp. 922-927.

[51] W. Maslak and B. H. Butkiewicz: Autonomous Control with Fuzzy Logic, Proc. of IEEE SPS, 2013, pp $\mathrm{xxx}$

[52] T. Zoubir and T. Abdelouaheb: A Fast Edge Detection Using Fuzzy Rules, Faculty of Engineering Science, Computer Science Department, Badji Mokhtar University Annaba, Algeria.

[53] T. M. Cabreira, G. P. Dimuro, and M. S. de Aguiar: An Evolutionary Learning Approach for Path Planning with Fuzzy Obstacle Detection and Avoidance in a Multi-Agent Environment, in IEEE third Brazilian Workshop on Social Simulation, Brazil, 2012. pp. 60-67.

\section{Authors' Profiles}



Ishaya Emmanuel received his B.Sc. degree from Usmanu Danfodiyo University Sokoto in 2012, and currently pursuing his M.Sc. at the same University.

His research area, for now, is control systems under artificial intelligence. His interests are mainly fuzzy controller design and implementation for autonomous/intelligent systems. Right now, his working on his dissertation to be soon completed and hope to be published. He is currently not affiliated to any institution, whether research or academics, but hope to soon get one so he could live up to his dream of research and knowledge transmission.

How to cite this paper: Ishaya Emmanuel,"Fuzzy Logic-Based Control for Autonomous Vehicle: A Survey", International Journal of Education and Management Engineering(IJEME), Vol.7, No.2, pp.41-49, 2017.DOI: 10.5815/ijeme.2017.02.05 